\title{
Unparticle physics and lepton flavor violating radion decays in the Randall-Sundrum scenario
}

\author{
E. O. Iltan, * \\ Physics Department, Middle East Technical University \\ Ankara, Turkey
}

\begin{abstract}
We predict the branching ratios of the lepton flavor violating radion decays $r \rightarrow e^{ \pm} \mu^{ \pm}$, $r \rightarrow e^{ \pm} \tau^{ \pm}$and $r \rightarrow \mu^{ \pm} \tau^{ \pm}$in the framework of the Randall-Sundrum scenario that the lepton flavor violation is carried by the scalar unparticle mediation. We observe that their BRs are strongly sensitive to the unparticle scaling dimension and, for its small values, the branching ratios can reach to the values of the order of $10^{-8}$, for the heavy lepton flavor case.
\end{abstract}

${ }^{*}$ E-mail address: eiltan@newton.physics.metu.edu.tr 


\section{Introduction}

Recently, Georgi [1, 2] proposed unparticle stuff which has non-integer scaling dimension $d_{u}$ and looks like a number of $d_{u}$ massless invisible particles. The idea behind is the existence of a non-trivial scale invariant sector beyond the standard model (SM), with non-trivial infrared fixed point and the scaling dimension $d_{u}$. Georgi suggested that, at the energy scales around $\Lambda_{U} \sim 1 \mathrm{TeV}$, this sector appears as so called unparticle stuff. The effective lagrangian can be constructed to describe the interactions of unparticles with the SM fields in the low energy level and this approach opens a window to test the effects of the possible scale invariant sector, experimentally.

The missing energies at various processes which can be measured at LHC or $e^{+} e^{-}$colliders, the dipole moments of fundamental particles and the processes in which the unparticle(s) appears as mediator are the possible candidates in order to search the effects of unparticle(s). There is various phenomenological work done in the literature on unparticles [2]-[74]: their effects on the missing energy of many processes, the anomalous magnetic moments, the electric dipole moments, $D^{0}-\bar{D}^{0}$ and $B^{0}-\bar{B}^{0}$ mixing, lepton flavor violating interactions, direct $\mathrm{CP}$ violation in particle physics; the phenomenological implications in cosmology and in astrophysics.

In this work, we study the lepton flavor violating (LFV) decays of the Randall Sundrum (RS1) radion field in the case that the LF violation 1 is carried by the scalar unparticle mediation. The RS1 model is based on the non-factorizable geometry [78, 79] in the five space-time dimensions and the extra dimension is compactified into $S^{1} / Z_{2}$ orbifold with two $4 \mathrm{D}$ brane boundaries. In one of the boundary, the so called Planck brane, the gravity is localized and in the another one, the TeV brane, all other fields are restricted. The size of extra dimension is proportional to the vacuum expectation of a scalar field and its fluctuation over the expectation value is called the radion field that has been studied extensively in the literature [80]-[93]. Here, we predict the BRs of the LFV decays in the framework of the RS1 scenario, by using the effective lagrangian in order to insert the possible scalar unparticle mediation. We observe that the BRs of the processes we study are strongly sensitive to the unparticle scaling dimension $d_{u}$ and, for its small values $d_{u}<1.1$, the BRs enhance considerably.

The paper is organized as follows: In Section 2, we present the the effective lagrangian and effective vertices which drive the LFV decays with scalar unparticle mediation, by respecting

\footnotetext{
${ }^{1}$ In the SM with massive neutrinos, so called $\nu \mathrm{SM}$ [75- [77, the lepton mixing mechanism is permitted. However, the negligibly small branching ratios (Brs) of LFV decays stimulate one to search for new LF violation mechanisms. In the present work, we do not take into account the LF violation coming from the possible massive neutrinos.
} 
the RS1 scenario. Furthermore, we give the expression for their BRs. Section 3 is devoted to the discussion and to our conclusions. In the appendix, we present the interaction vertices including the radion field.

\section{The LFV RS1 radion decay with scalar unparticle me- diation}

The LFV processes are among the rare decays in the sense that they exist at least in the one loop level and their BRs are small. However, their existence in the loop level makes them worthwhile to analyze since physical quantities related to them contain considerable information about the model used and the free parameters existing. In the present work, we study the LFV decays of the radion field, in the framework of the RS1 scenario. Here we assume that the LF violation is carried by the unparticle stuff which is introduced by Georgi [1, 2]. The starting point of the idea is the interaction of two sectors, the SM and the ultraviolet sector with nontrivial infrared fixed point, at high energy level. The ultraviolet sector appears as new degrees of freedom, called unparticles, being massless and having non integral scaling dimension $d_{u}$ around, $\Lambda_{U} \sim 1 T e V$. This mechanism results in the existence of the effective field theory with effective Lagrangian in the low energy level and the corresponding Lagrangian reads

$$
\mathcal{L}_{e f f} \sim \frac{\eta}{\Lambda_{U}^{d_{u}+d_{S M}-n}} O_{S M} O_{U}
$$

where $O_{U}$ is the unparticle operator, the parameter $\eta$ is related to the energy scale of ultraviolet sector, the low energy one and the matching coefficient [1, 2, 3] and $n$ is the space-time dimension.

At this stage, we would like to give a brief explanation about the RS1 scenario and the effective Lagrangian which is responsible for the LFV decay underconsideration. The RS1 model is formulated in the warped extra dimension, which is compactified into $S^{1} / Z_{2}$ orbifold. There exist two 4D surfaces (branes), which are boundaries of the extra dimension, in 5D world. One of the brane is called the Planck brane where the gravity, extending into the bulk with varying strength, peaks near and the other one is called the TeV brane where we live. The considered behavior of the gravity results in the explanation of the well known hierarchy problem. In addition to this, the cosmological constant problem is solved with the help of the 
equal and opposite tensions in these two branes. The background metric of this 5D world is

$$
d s^{2}=e^{-2 A(y)} \eta_{\mu \nu} d x^{\mu} d x^{\nu}-d y^{2}
$$

where $A(y)=k|y|, k$ is the bulk curvature constant, $y$ is the extra dimension parametrized as $y=R \theta$. The exponential factor $e^{-k L}$ with the inter brane separation $L=R \pi$, is the warp factor which ensures that all the mass terms are rescaled in the TeV brane. With a rough estimate $L \sim 30 / k$ the hierarchy problem is solved and all mass terms are pulled down to the $\mathrm{TeV}$ scale. In this scenario, the radion field appears as fluctuation over the expectation value of the field $L(x)$ that its vacuum expectation value is related to the size $L$ of extra dimension. On the other hand, the field $L(x)$ should acquire a mass not to have a conflict with the equivalence principle and, the radion field can be stabilized with a mechanism, proposed by Goldberger and Wise [80]. Including the radial fluctuations, the metric in 5D is defined as [81]

$$
d s^{2}=e^{-2 A(y)-2 F(x)} \eta_{\mu \nu} d x^{\mu} d x^{\nu}-\left(1+2 F(x) d y^{2}\right.
$$

where $F(x)$ the scalar field,

$$
F(x)=\frac{1}{\sqrt{6} M_{P l} e^{-k L}} r(x),
$$

and $r(x)$ is the normalized radion field (see for example [82]). Finally, the the induced metric at the orbifold point $\theta=\pi$ ( $\mathrm{TeV}$ brane) reads,

$$
g_{\mu \nu}^{i n d}=e^{-2 A(L)-2 \frac{\gamma}{v} r(x)} \eta_{\mu \nu}
$$

with $\gamma=\frac{v}{\sqrt{6} \Lambda_{r}}, \Lambda_{r}=M_{P l} e^{-k L}$ and $v$ is the vacuum expectation value of the SM Higgs boson.

Here, we are ready the construct the effective interaction lagrangian for the LFV decays we study. Notice that we choose the appropriate operators with lowest possible dimension since they have the most powerful effect in the low energy effective theory (see for example [12]). The part of the effective lagrangian which is responsible for the LF violation reads

$$
\mathcal{L}_{1}=\frac{\sqrt{-g^{i n d}}}{\Lambda^{d u-1}}\left(\lambda_{i j}^{S} \bar{l}_{i} l_{j}+\lambda_{i j}^{P} \bar{l}_{i} i \gamma_{5} l_{j}\right) O_{U},
$$

where $l$ is the lepton field, $O_{U}$ is the scalar unparticle $(U)$ operator and $\lambda_{i j}^{S}\left(\lambda_{i j}^{P}\right)$ is the scalar (pseudoscalar) coupling. In addition to the tree level $U-l_{1}-l_{2}$ interaction with the coupling $\sim \frac{1}{\Lambda^{d u-1}}\left(\lambda_{i j}^{S}+i \lambda_{i j}^{P} \gamma_{5}\right)$, the lagrangian in eq. (6) drives the four point $r-U-l_{1}-l_{2}$ interaction

\footnotetext{
${ }^{2}$ The $5 \mathrm{D}$ cosmological constant does not vanish, however, the low energy effective theory has flat 4D spacetime by considering both branes have equal and opposite tensions.
} 
due to the factor $\sqrt{-g^{i n d}}=e^{-4 A(L)-4 \frac{\gamma}{v} r(x)}$ (see Fig. 1-a). Here the background term $e^{-4 A(L)}$ in $\sqrt{-g^{i n d}}$ is embedded into the redefinitions of the fields on the $\mathrm{TeV}$ brane, namely, they are warped as $l \rightarrow e^{3 A(L) / 2} l_{\text {warp }}, \frac{O_{U}}{\Lambda^{d u-1}} \rightarrow e^{A(L)}\left(\frac{O_{U}}{\Lambda^{d u-1}}\right)_{\text {warp }}$, and in the following we use warped fields without the warp index. Since the FV radion decays, $r \rightarrow l_{1}^{-} l_{2}^{+}$, can exist at least in one loop level (see Fig 2), one needs the scalar unparticle propagator which is obtained by using the scale invariance. The two point function of the unparticle results in [2, 4]

$$
\int d^{4} x e^{i p x}<0 \mid T\left(O_{U}(x) O_{U}(0)\right) 0>=i \frac{A_{d_{u}}}{2 \pi} \int_{0}^{\infty} d s \frac{s^{d_{u}-2}}{p^{2}-s+i \epsilon}=i \frac{A_{d_{u}}}{2 \sin \left(d_{u} \pi\right)}\left(-p^{2}-i \epsilon\right)^{d_{u}-2}
$$

with the factor $A_{d_{u}}$

$$
A_{d_{u}}=\frac{16 \pi^{5 / 2}}{(2 \pi)^{2 d_{u}}} \frac{\Gamma\left(d_{u}+\frac{1}{2}\right)}{\Gamma\left(d_{u}-1\right) \Gamma\left(2 d_{u}\right)} .
$$

The function $\frac{1}{\left(-p^{2}-i \epsilon\right)^{2-d_{u}}}$ in eq. (17) becomes

$$
\frac{1}{\left(-p^{2}-i \epsilon\right)^{2-d_{u}}} \rightarrow \frac{e^{-i d_{u} \pi}}{\left(p^{2}\right)^{2-d_{u}}}
$$

for $p^{2}>0$ and a non-trivial phase appears as a result of non-integral scaling dimension.

On the other hand, the part of the lagrangian which carries the interaction of leptons with the radion field reads (for example see [83, 84])

$$
\mathcal{L}_{2}=\sqrt{-g^{i n d}}\left(g^{i n d \mu \nu} \bar{l} \gamma_{\mu} i D_{\nu} l-m_{l} \bar{l} l\right),
$$

where

$$
D_{\mu} l=\partial_{\mu} l+\frac{1}{2} w_{\mu}^{a b} \Sigma_{a b} l
$$

and $\Sigma_{a b}=\frac{1}{4}\left[\gamma_{a}, \gamma_{b}\right]$. Here $w_{\mu}^{a b}$ is the spin connection which reads

$$
w_{\mu}^{a b}=-\frac{\gamma}{v} \partial_{\nu} r\left(e^{\nu b} e_{\mu}^{a}-e^{\nu a} e_{\mu}^{b}\right)
$$

linear in $r$. In this equation, $e_{\mu}^{a}$ are the vierbein fields and they satisfy the relation

$$
e_{a}^{\mu} e^{a \nu}=g^{i n d \mu \nu}
$$

Using eqs. (10)-(13), one gets the part of the lagrangian which drives the tree level $l-l-r$ interaction (see Fig.1-b) as

$$
\mathcal{L}^{\prime}{ }_{2}=\left\{-3 \frac{\gamma}{v} r \bar{l} i \not \partial l-3 \frac{\gamma}{2 v} \bar{l} i \not \partial r l+4 \frac{\gamma}{v} m_{l} r \bar{l} l\right\} .
$$


Now, we present the matrix element square of the LFV radion decay which exits at least in one loop order (see Fig. 2 for the possible vertex and self energy diagrams):

$$
|M|^{2}=2\left(m_{r}^{2}-\left(m_{l_{1}^{-}}+m_{l_{2}^{+}}\right)^{2}\right)|A|^{2}+2\left(m_{r}^{2}-\left(m_{l_{1}^{-}}-m_{l_{2}^{+}}\right)^{2}\right)\left|A^{\prime}\right|^{2},
$$

where

$$
\begin{aligned}
A & =\int_{0}^{1} d x f_{\text {self }}^{S}+\int_{0}^{1} d x \int_{0}^{1-x} d y f_{\text {vert }}^{S}, \\
A^{\prime} & =\int_{0}^{1} d x f_{\text {self }}^{\prime S}+\int_{0}^{1} d x \int_{0}^{1-x} d y f_{\text {vert }}^{\prime S},
\end{aligned}
$$

and the explicit expressions of $f_{\text {self }}^{S}, f_{\text {self }}^{\prime S}, f_{\text {vert }}^{S}, f_{\text {vert }}^{\prime S}$ read

$$
\begin{aligned}
& f_{\text {self }}^{S}=\frac{i c_{1}(1-x)^{1-d_{u}}}{32 \pi^{2}\left(m_{l_{2}^{+}}-m_{l_{1}^{-}}\right)\left(1-d_{u}\right)} \sum_{i=1}^{3}\left\{\left(\lambda_{i l_{1}}^{S} \lambda_{i l_{2}}^{S}+\lambda_{i l_{1}}^{P} \lambda_{i l_{2}}^{P}\right)(1-x)\right. \\
& \times\left(m_{l_{1}^{-}}\left(5 m_{l_{1}^{-}}-3 m_{l_{2}^{+}}\right) L_{\text {self }}^{d_{u}-1}-m_{l_{2}^{+}}\left(5 m_{l_{2}^{+}}-3 m_{l_{1}^{-}}\right) L_{\text {self }}^{\prime d_{u}-1}\right) \\
& \left.+\left(\lambda_{i l_{1}}^{P} \lambda_{i l_{2}}^{P}-\lambda_{i l_{1}}^{S} \lambda_{i l_{2}}^{S}\right) m_{i}\left(\left(3 m_{l_{2}^{+}}-5 m_{l_{1}^{-}}\right) L_{\text {self }}^{d_{u}-1}-\left(3 m_{l_{1}^{-}}-5 m_{l_{2}^{+}}\right) L_{s e l f}^{\prime d_{u}-1}\right)\right\}, \\
& f_{\text {self }}^{\prime S}=\frac{\left.c_{1}(1-x)^{1-d_{u}}\right]}{32 \pi^{2}\left(m_{l_{2}^{+}}+m_{l_{1}^{-}}\right)\left(1-d_{u}\right)} \sum_{i=1}^{3}\left\{\left(\lambda_{i l_{1}}^{S} \lambda_{i l_{2}}^{P}-\lambda_{i l_{1}}^{P} \lambda_{i l_{2}}^{S}\right)(1-x)\right. \\
& \times\left(m_{l_{1}^{-}}\left(3 m_{l_{2}^{+}}+5 m_{l_{1}^{-}}\right) L_{\text {self }}^{d_{u}-1}-m_{l_{2}^{+}}\left(3 m_{l_{1}^{-}}+5 m_{l_{2}^{+}}\right) L_{\text {self }}^{\prime d_{u}-1}\right) \\
& \left.\left.+\left(\lambda_{i l_{1}}^{S} \lambda_{i l_{2}}^{P}+\lambda_{i l_{1}}^{P} \lambda_{i l_{2}}^{S}\right) m_{i}\left(\left(3 m_{l_{2}^{+}}+5 m_{l_{1}^{-}}\right) L_{\text {self }}^{d_{u}-1}+\left(3 m_{l_{1}^{-}}+5 m_{l_{2}^{+}}\right) L_{\text {self }}^{\prime d_{u}-1}\right)\right)\right\}, \\
& f_{\text {vert }}^{S}=\frac{-3 i c_{1}(1-x-y)^{1-d_{u}}}{32 \pi^{2}} \sum_{i=1}^{3} \frac{1}{L_{\text {vert }}^{2-d_{u}}}\left\{\left(\lambda_{i l_{1}}^{S} \lambda_{i l_{2}}^{S}+\lambda_{i l_{1}}^{P} \lambda_{i l_{2}}^{P}\right)\{(1-x-y)\right. \\
& \times\left(m_{l_{2}^{+}} m_{l_{1}^{-}}\left(m_{l_{2}^{+}}+m_{l_{1}^{-}}\right)(1-x-y+2 x y)+2\left(m_{l_{2}^{+}}^{3} y(y-1)+m_{l_{1}^{-}}^{3} x(x-1)\right)\right) \\
& -\frac{3 L_{v e r t}}{1-d_{u}}\left(m_{l_{1}^{-}}(2 x-1)+m_{l_{2}^{+}}(2 y-1)\right) \\
& \left.+m_{r}^{2}\left(m_{l_{1}^{-}} x(2 y-1)(x-1)+m_{l_{2}^{+}} y(2 x-1)(y-1)\right)\right) \\
& \left.+5 m_{i}^{2}\left(m_{l_{1}^{-}}(2 x-1)+m_{l_{2}^{+}}(2 y-1)\right)\right\} \\
& +\left(\lambda_{i l_{1}}^{P} \lambda_{i l_{2}}^{P}-\lambda_{i l_{1}}^{S} \lambda_{i l_{2}}^{S}\right)\left\{(1-x-y) m_{i}\left(2 m_{l_{1}^{-}} m_{l_{2}^{+}}+m_{l_{1}^{-}}^{2}(4 x-3)+m_{l_{2}^{+}}^{2}(4 y-3)\right)\right. \\
& \left.\left.-\frac{8 m_{i}}{1-d_{u}} L_{v e r t}-m_{i} m_{r}^{2}(3 x+3 y-4 x y)+8 m_{i}^{3}\right\}\right\} \text {, }
\end{aligned}
$$




$$
\begin{aligned}
f_{v e r t}^{\prime S} & =\frac{-3 c_{1}(1-x-y)^{1-d_{u}}}{32 \pi^{2}} \sum_{i=1}^{3} \frac{1}{L_{v e r t}^{2-d_{u}}}\left\{\left(\lambda_{i l_{1}}^{P} \lambda_{i l_{2}}^{S}-\lambda_{i l_{1}}^{S} \lambda_{i l_{2}}^{P}\right)\{(1-x-y)\right. \\
& \times\left(m_{l_{2}^{+}} m_{l_{1}^{-}}\left(m_{l_{2}^{+}}-m_{l_{1}^{-}}\right)(1-x-y+2 x y)+2\left(-m_{l_{2}^{+}}^{3} y(y-1)+m_{l_{1}^{-}}^{3} x(x-1)\right)\right) \\
& -\frac{3 L_{v e r t}}{1-d_{u}}\left(m_{l_{1}^{-}}(2 x-1)-m_{l_{2}^{+}}(2 y-1)\right) \\
& \left.+m_{r}^{2}\left(m_{l_{1}^{-}} x(2 y-1)(x-1)-m_{l_{2}^{+}} y(2 x-1)(y-1)\right)\right) \\
& \left.+5 m_{i}^{2}\left(m_{l_{1}^{-}}(2 x-1)-m_{l_{2}^{+}}(2 y-1)\right)\right\} \\
& +\left(\lambda_{i l_{1}}^{S} \lambda_{i l_{2}}^{P}+\lambda_{i l_{1}}^{P} \lambda_{i l_{2}}^{S}\right)\left\{(1-x-y) m_{i}\left(-2 m_{l_{1}^{-}} m_{l_{2}^{+}}+m_{l_{1}^{-}}^{2}(4 x-3)+m_{l_{2}^{+}}^{2}(4 y-3)\right)\right. \\
& \left.\left.-\frac{8 m_{i}}{1-d_{u}} L_{v e r t}-m_{i} m_{r}^{2}(3 x+3 y-4 x y)+8 m_{i}^{3}\right\}\right\},
\end{aligned}
$$

with

$$
\begin{aligned}
L_{\text {self }} & =x\left(m_{l_{1}^{-}}^{2}(1-x)-m_{i}^{2}\right), \\
L_{\text {self }}^{\prime} & =x\left(m_{l_{2}^{+}}^{2}(x-1)-m_{i}^{2}\right), \\
L_{\text {vert }} & =\left(m_{l_{1}^{-}}^{2} x+m_{l_{2}^{+}}^{2} y\right)(1-x-y)-m_{i}^{2}(x+y)+m_{r}^{2} x y,
\end{aligned}
$$

and

$$
c_{1}=\frac{\gamma A_{d_{u}}}{2 v \sin \left(d_{u} \pi\right) \Lambda_{u}^{2\left(d_{u}-1\right)}} .
$$

In eq. (17), the flavor changing scalar and pseudoscalar couplings $\lambda_{i l_{1(2)}}^{S, P}$ represent the effective interaction between the internal lepton $i,(i=e, \mu, \tau)$ and the outgoing $l_{1}^{-}\left(l_{2}^{+}\right)$lepton (anti lepton).

Finally, the BR for $r \rightarrow l_{1}^{-} l_{2}^{+}$can be obtained by using the matrix element square as

$$
B R\left(r \rightarrow l_{1}^{-} l_{2}^{+}\right)=\frac{1}{16 \pi m_{r}} \frac{|M|^{2}}{\Gamma_{r}},
$$

with the radion total decay width $\Gamma_{r}$. In the numerical analysis, we consider the BR due to the production of sum of charged states, namely,

$$
B R\left(r \rightarrow l_{1}^{ \pm} l_{2}^{ \pm}\right)=\frac{\Gamma\left(r \rightarrow\left(\bar{l}_{1} l_{2}+\bar{l}_{2} l_{1}\right)\right)}{\Gamma_{r}}
$$

\section{Discussion}

In this section, we analyze the LFV RS1 radion decays $r \rightarrow l_{1}^{-} l_{2}^{+}$in the case that the flavor violation is carried by the scalar unparticle mediation and we estimate their BRs. The LFV 
radion decays $r \rightarrow l_{1}^{-} l_{2}^{+}$can exist at least in one loop level and, in the present case, the flavor violation is driven by the fermion-fermion- $U$ couplings, in the effective theory. The scaling dimension of the unparticle, the couplings, the radion mass and the energy scale is the free parameters of the scenario studied. At this stage, we discuss our restrictions for these free parameters:

- The scaling dimension $d_{u}$ is chosen in the range $1<d_{u}<2$. For $d_{u}>1$, one is free from the non-integrable singularity problem in the decay rate [2]. On the other hand the momentum integrals converges for $d_{u}<2[20]$.

- Here we consider that the diagonal couplings $\lambda_{i i}$ respects the lepton family the hierarchy, $\lambda_{\tau \tau}>\lambda_{\mu \mu}>\lambda_{e e}$, and the off-diagonal couplings, $\lambda_{i j}, i \neq j$ are family blind and universal. Furthermore, we take the off diagonal couplings as, $\lambda_{i j}=\kappa \lambda_{e e}$ with $\kappa<1$. In our numerical calculations, we choose $\kappa=0.5$ and take the greatest numerical value of diagonal couplings not more than the order of one.

- For the mass of the radion, we choose the values $m_{r}=200(\mathrm{GeV}), m_{r}=500(\mathrm{GeV})$ and $m_{r}=800(\mathrm{GeV})$ to observe the radion mass dependence of the BRs of the decays under consideration.

- We take the energy scale at least $\Lambda_{u}=10(\mathrm{TeV})$ and study $\Lambda_{u}$ dependence of the BRs for its various values.

For the calculation of the BRs of $r \rightarrow l_{1}^{-} l_{2}^{+}$decays we need the total decay width $\Gamma_{r}$ of the radion and we use the theoretical predictions given in the literature. The decay width is dominated by $r \rightarrow g g$ for the radion mass $m_{r} \leq 150 \mathrm{GeV}$. This quantity is calculated from the trace anomaly which exists in one loop order. For the higher masses of the radion, which are beyond the WW and ZZ thresholds, the main decay mode is $r \rightarrow W W$. In this work, we take the total decay width $\Gamma_{r}$ of the radion by considering the dominant decays $r \rightarrow g g\left(\gamma \gamma, f f, W^{+} W^{-}, Z Z, S S\right)$ where $S$ are the neutral Higgs particles (see [87] for the explicit expressions of these decay widths). Notice that we include the possible processes in the $\Gamma_{r}$ according to the mass of the radion, in our calculations. Throughout our calculations we use the input values given in Table (11). 


\begin{tabular}{|l|l|}
\hline Parameter & Value \\
\hline \hline$m_{e}$ & $0.0005(\mathrm{GeV})$ \\
$m_{\mu}$ & $0.106(\mathrm{GeV})$ \\
$m_{\tau}$ & $1.780(\mathrm{GeV})$ \\
\hline
\end{tabular}

Table 1: The values of the input parameters used in the numerical calculations.

In Fig 3, we present the contribution of the scalar unparticle to the $\operatorname{BR}\left(r \rightarrow \mu^{ \pm} e^{ \pm}\right)$with respect to the scale parameter $d_{u}$, for the energy scale $\Lambda_{u}=10 \mathrm{TeV}$, the couplings $\lambda_{e e}=0.01$ and $\lambda_{\mu \mu}=0.1$. Here the solid (dashed, small dashed) line represents the BR for $m_{r}=200(\mathrm{GeV})$ $\left(m_{r}=500(\mathrm{GeV}), m_{r}=800(\mathrm{GeV})\right)$. The BR of the decay considered is strongly sensitive to the scale $d_{u}$ and, with the decreasing values of $d_{u}$, there is a considerable enhancement in the $\mathrm{BR}$. The BR reaches to the numerical values $10^{-10}$, for $d_{u}<1.1$ and $m_{r}=200(\mathrm{GeV})$. For the heavier mass values of the radion the BR is suppressed and it decreases to the values of the order of $10^{-12}$ for $d_{u}<1.1$ and $m_{r}=500,800(\mathrm{GeV})$.

In Fig, 4 , we show the contribution of the scalar unparticle to the BR $\left(r \rightarrow \tau^{ \pm} e^{ \pm}\right)$with respect to the scale parameter $d_{u}$, for the energy scale $\Lambda_{u}=10 \mathrm{TeV}$, the couplings $\lambda_{e e}=0.01$ and $\lambda_{\tau \tau}=1$. Here the solid (dashed, small dashed) line represents the BR for $m_{r}=200(\mathrm{GeV})$ $\left(m_{r}=500(\mathrm{GeV}), m_{r}=800(\mathrm{GeV})\right)$. For $d_{u}<1.1$ the BR increases considerably and it is in the range $10^{-10}-10^{-6}$, for $m_{r}=200(\mathrm{GeV})$. For the radion masses $m_{r}=500,800(\mathrm{GeV})$, the BR is suppressed to the values $10^{-12}$ for $d_{u} \sim 1.1$.

Fig 5 represents the contribution of the scalar unparticle to the $\operatorname{BR}\left(r \rightarrow \tau^{ \pm} \mu^{ \pm}\right)$with respect to the scale parameter $d_{u}$, for the energy scale $\Lambda_{u}=10 \mathrm{TeV}$, the couplings $\lambda_{\mu \mu}=0.1$ and $\lambda_{\tau \tau}=1$. Here the solid (dashed, small dashed) line represents the BR for $m_{r}=200(\mathrm{GeV})$ $\left(m_{r}=500(\mathrm{GeV}), m_{r}=800(\mathrm{GeV})\right)$. The BR for $d_{u}<1.1$ and $m_{r}=200(\mathrm{GeV})$ is large similar to the $\left(r \rightarrow \tau^{ \pm} e^{ \pm}\right)$decay, and it is in the range of $10^{-10}-10^{-6}$, in this region of the scale dimension.

These figures show that the BRs of the LFV decays are sensitive to the scaling dimension $d_{u}$ and they become negligibly small (quite large) for the values of the scaling dimension $d_{u}$ greater and far from 1.1 (near to 1.0).

Now, we would like to analyze the energy scale $\Lambda_{u}$ and the parameter $\lambda$ dependence of the BRs of the LFV decays in the various figures, for completeness.

Fig. 6] (77, 8) is devoted to the contribution of the scalar unparticle to the BR $\left(r \rightarrow \mu^{ \pm} e^{ \pm}\right)$ $\left(\mathrm{BR}\left(r \rightarrow \tau^{ \pm} e^{ \pm}\right), \mathrm{BR}\left(r \rightarrow \tau^{ \pm} \mu^{ \pm}\right)\right)$with respect to the energy scale $\Lambda_{u}$, for $m_{r}=200(\mathrm{GeV})$ and the couplings $\lambda_{e e}=0.01$ and $\lambda_{\mu \mu}=0.1\left(\lambda_{e e}=0.01\right.$ and $\lambda_{\tau \tau}=1.0, \lambda_{\mu \mu}=0.1$ and $\left.\lambda_{\tau \tau}=1.0\right)$. 
Here the solid-dashed-small dashed lines represent the BR for $d_{u}=1.1-d_{u}=1.2-d_{u}=1.5$. The increasing values of the energy scale $\Lambda_{u}$ result in the suppression in the BR and the numerical values of the order of $10^{-13}\left(0.510^{-10}, 0.510^{-10}\right)$ can be reached for the energy scale $\Lambda_{u}, \Lambda_{u}<10(\mathrm{TeV})$ and $d_{u}=1.1$. This figure shows also the scale $d_{u}$ sensitivity of the BRs of the decays under consideration.

Fig. 9 (10, 11) represents the the contribution of the scalar unparticle to the $\mathrm{BR}\left(r \rightarrow \mu^{ \pm} e^{ \pm}\right)$ $\left(\mathrm{BR}\left(r \rightarrow \tau^{ \pm} e^{ \pm}\right), \mathrm{BR}\left(r \rightarrow \tau^{ \pm} \mu^{ \pm}\right)\right)$with respect to the parameter $\lambda$, for the energy scale $\Lambda_{u}=10 \mathrm{TeV}$ and the radion mass $m_{r}=200(\mathrm{GeV})$. Here, we consider that the parameter $\lambda$ is connected to the couplings by the equalities, $\lambda_{e e}=\lambda, \lambda_{\mu \mu}=10 \lambda, \lambda_{\tau \tau}=100 \lambda$. The soliddashed-small dashed lines represent the BR for $d_{u}=1.1-d_{u}=1.2-d_{u}=1.5$. It is observed that the BR is strongly sensitive to the parameter $\lambda$ as expected and, its numerical value is of the order of $10^{-13}\left(0.510^{-10}, 0.510^{-10}\right)$ for $\lambda \sim 0.01$, for the scaling dimension $d_{u}=1.1$.

As a summary, the LFV decays of the radion in the RS1 model are strongly sensitive to the unparticle scaling dimension and, for its small values $d_{u}<1.1$, the BRs enhance considerably. The other free parameters of the scenario studied are the $U$-fermion-fermion couplings, the energy scale and the radion mass and the dependencies of the BRs of LFV decays to these free parameters are also strong. The possible production of the radion (the most probable production is due to the gluon fusion, $g g \rightarrow r$ [87]) would stimulate one to study its LFV decays and the forthcoming experimental results would be instructive in order to test the possible signals coming from the extra dimensions and the new physics which drives the flavor violation, here is the unparticle physics.

\section{A The vertices including the radion field, in the present work}

In this section we present the vertices, including the radion field, used in our calculations. 


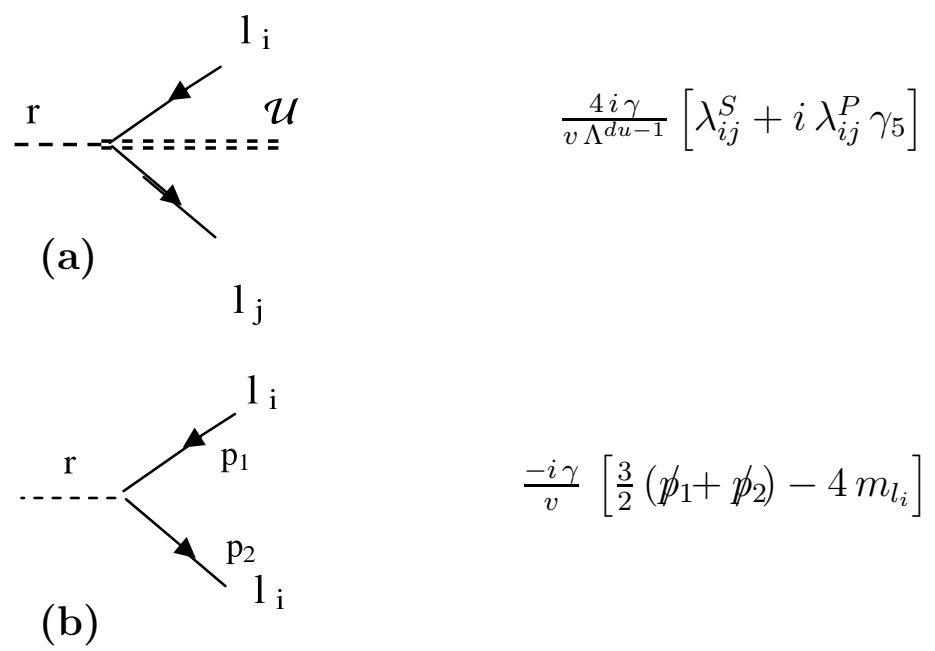

Figure 1: The vertices including the radion field. 


\section{References}

[1] H. Georgi, Phys. Rev. Lett. 98, 221601 (2007).

[2] H. Georgi, Phys. Lett. B650, 275 (2007).

[3] R. Zwicky, hep-ph/0707.0677 (2007).

[4] K. Cheung, W. Y. Keung and T. C. Yuan, Phys. Rev. Lett. 99, 051803 (2007).

[5] M. X. Luo and G. H. Zhu, hep-ph/0704.3532 (2007).

[6] M. A. Stephanov, Phys. Rev. D 76, 035008 (2007).

[7] K. Cheung, W. Y. Keung and T. C. Yuan, hep-ph/0706.3155 (2007).

[8] M. X. Luo, W. Wu and G. H. Zhu, hep-ph/0708.0671 (2007).

[9] C. H. Chen and C. Q. Geng, hep-ph/0705.0689 (2007).

[10] C. H. Chen and C. Q. Geng, Phys. Rev. D76, 036007 (2007).

[11] C. H. Chen and C. Q. Geng, hep-ph/0709.0235 (2007).

[12] S. L. Chen and X. G. He, hep-ph/0705.3946 (2007).

[13] T. M. Aliev, A. S. Cornell and N. Gaur, hep-ph/0705.1326 (2007).

[14] T. M. Aliev, A. S. Cornell and N. Gaur, JHEP 07, 072 (2007).

[15] T.M. Aliev, M. Savci hep-ph/0710.1505 (2007)

[16] G. J. Ding and M. L. Yan, hep-ph/0705.0794 (2007).

[17] G. J. Ding and M. L. Yan, hep-ph/0706.0325 (2007).

[18] X. Q. Li and Z. T. Wei, Phys. Lett. B651, 380 (2007).

[19] X. Q. Li and Z. T. Wei, hep-ph/0707.2285 (2007).

[20] Y. Liao, hep-ph/0705.0837 (2007).

[21] Y. Liao, hep-ph/0708.3327 (2007).

[22] Y. Liao and J. Y. Liu, hep-ph/0706.1284 (2007). 
[23] P. J. Fox, A. Rajaraman and Y. Shirman, hep-ph/0705.3092 (2007).

[24] S. Catterall and F. Sannino, Phys. Rev. D76, 034504 (2007).

[25] C. D. Lu, W. Wang and Y. M. Wang, hep-ph/0705.2909 (2007).

[26] N. Greiner, hep-ph/0705.3518 (2007).

[27] D. Choudhury, D. K. Ghosh and Mamta, hep-ph/0705.3637 (2007).

[28] H. Davoudiasl, hep-ph/0705.3636 (2007).

[29] S. L. Chen, X. G. He and H. C. Tsai, hep-ph/0707.0187 (2007).

[30] P. Mathews and V. Ravindran, hep-ph/0705.4599 (2007).

[31] S. Zhou, hep-ph/0706.0302 (2007).

[32] R. Foadi, M. T. Frandsen, T. A. Ryttov and F. Sannino, hep-ph/0706.1696 (2007).

[33] M. Bander, J. L. Feng, A. Rajaraman and Y. Shirman, hep-ph/0706.2677 (2007).

[34] T. G. Rizzo, hep-ph/0706.3025 (2007).

[35] H. Goldberg and P. Nath, hep-ph/0706.3898 (2007).

[36] T. Kikuchi and N. Okada, hep-ph/0707.0893 (2007).

[37] R. Mohanta and A. K. Giri, hep-ph/0707.1234 (2007).

[38] R. Mohanta and A. K. Giri, hep-ph/0707.3308 (2007).

[39] C. S. Huang and X. H. Wu, hep-ph/0707.1268 (2007).

[40] N. V. Krasnikov, hep-ph/0707.1419 (2007).

[41] A. Lenz, hep-ph/0707.1535 (2007).

[42] D. Choudhury and D. K. Ghosh, hep-ph/0707.2074 (2007).

[43] H. Zhang, C. S. Li and Z. Li, hep-ph/0707.2132 (2007).

[44] Y. Nakayama, hep-ph/0707.2451 (2007).

[45] N. G. Deshpande, X. G. He and J. Jiang, hep-ph/0707.2959 (2007). 
[46] N. G. Deshpande, S. D. H. Hsu and J. Jiang, hep-ph/0708.2735 (2007).

[47] A. Delgado, J. R. Espinosa and M. Quiros, hep-ph/0707.4309 (2007).

[48] M. Neubert, hep-ph/0708.0036 (2007).

[49] S. Hannestad, G. Raffelt and Y. Y. Y. Wong, hep-ph/0708.1404 (2007).

[50] P. K. Das, hep-ph/0708.2812 (2007).

[51] S. Das, S. Mohanty and K. Rao, hep-ph/0709.2583 (2007).

[52] G. Bhattacharyya, D. Choudhury and D. K. Ghosh, hep-ph/0708.2835 (2007).

[53] D. Majumdar, hep-ph/0708.3485 (2007).

[54] A. T. Alan and N. K. Pak, hep-ph/0708.3802 (2007).

[55] A. Freitas and D. Wyler, hep-ph/0708.4339 (2007).

[56] I. Gogoladze, N. Okada and Q. Shafi, hep-ph/0708.4405 (2007).

[57] T. i. Hur, P. Ko and X. H. Wu, hep-ph/0709.0629 (2007).

[58] L. Anchordoqui and H. Goldberg, hep-ph/0709.0678 (2007).

[59] S. Majhi, hep-ph/0709.1960 (2007).

[60] J. McDonald, hep-ph/0709.2350 (2007).

[61] M. C. Kumar, P. Mathews, V. Ravindran and A. Tripathi, hep-ph/0709.2478 (2007).

[62] K. M. Cheung, W. Y. Keung, T. C. Yuan, hep-ph/0710.2230 (2007).

[63] A. Kobakhidze, hep-ph/0709.3782 (2007).

[64] G. J. Ding and M. L. Yan, hep-ph/0709.3435 (2007).

[65] A.B. Balantekin , K.O. Ozansoy, hep-ph/0710.0028 (2007).

[66] E. O. lltan, hep-ph/0710.2677 (2007).

[67] S. L. Chen, X. G. He, X. Q. Li, H. C. Tsai, Z. T. Wei, hep-ph/0710.3663 (2007).

[68] I. Lewis hep-ph/0710.4147 (2007). 
[69] A. T. Alan and N. K. Pak, hep-ph/0710.4239 (2007).

[70] G.L. Alberghi, A.Y. Kamenshchik, A. Tronconi, G.P. Vacca, G. Venturi, hep-th/0710.4275 (2007).

[71] R. Zwicky, hep-ph/0710.4430 (2007).

[72] S.-L. C., X. G. He, X. P. Hu, Y. Liao, hep-ph/0710.5129 (2007).

[73] O. Cakir, K.O. Ozansoy, hep-ph/0710.5773 (2007).

[74] T. Kikuchi, N. Okada, hep-ph/0711.1506 (2007).

[75] B. Pontecorvo, Zh. Eksp. Teor. Fiz. 33, 549 (1957).

[76] Z. Maki, M. Nakagawa, and S. Sakata, Prog. Theor. Phys. 28, 870 (1962).

[77] B. Pontecorvo, Sov. Phys. JETP 26, 984 (1968).

[78] L. Randall, R.Sundrum, Phys. Rev. Lett. 83 (1999) 3370.

[79] L. Randall, R.Sundrum, Phys. Rev. Lett. 83 (1999) 4690.

[80] W. D. Goldberger, M. B. Wise, Phys. Rev. Lett. D 83, 4922 (1999).

[81] C. Charmousis, R. Gregory, V. A. Rubakov, Phys. Rev. D 62, 067505 (2000).

[82] C. Csaki, M. L. Graesser, G. D. Gribs, Phys. Rev. D 63, 065002 (2001).

[83] P. K. Das, Int. J. Mod. Phys A21, 52052006.

[84] P. K. Das, Phys. Rev. D 72, 055009 (2005).

[85] G. D. Kribs, eConf C010630 P317 (2001), hep-ph/0110242

[86] T. Han, G. D. Kribs, B. McElrath Phys. Rev. D 64, 076003 (2001).

[87] K. Cheung, Phys. Rev. D 63, 056007 (2001).

[88] K. Cheung, hep-ph/0408200 2004.

[89] G. F. Giudice, R. Rattazzi, J. D. Wells, Nucl. Phys. B 595, 250 (2001).

[90] U. Mahanta, A. Datta Phys. Lett. B 483, 196 (2000). 
[91] K. Cheung, C. S. Kim, J. Song, Phys. Rev. D67, 075017 (2003).

[92] C. Csaki, hep-ph/0404096 2004.

[93] E.O. Iltan, B. Korutlu, hep-ph/0610147 (2006).

[94] C. Csaki, J. Hubisz, S. J. Lee, hep-ph/0705.3844 (2007). 


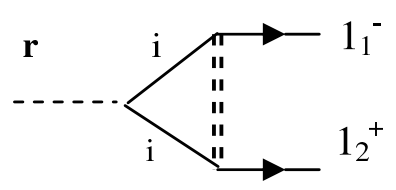

(a)

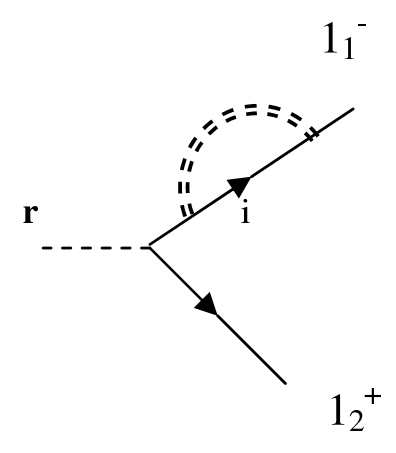

(b)

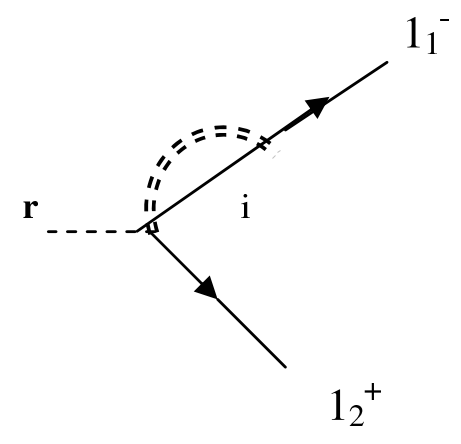

(d)

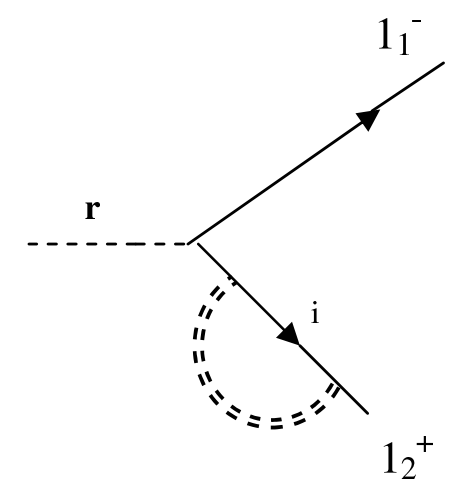

(c)

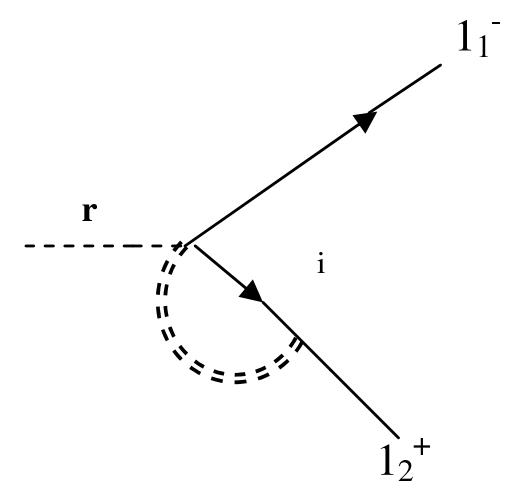

(e)

Figure 2: One loop diagrams contribute to $r \rightarrow l_{1}^{-} l_{2}^{+}$decay with scalar unparticle mediator. Solid line represents the lepton field: $i$ represents the internal lepton, $l_{1}^{-}\left(l_{2}^{+}\right)$outgoing lepton (anti lepton), dashed line the radion field, double dashed line the unparticle field. 


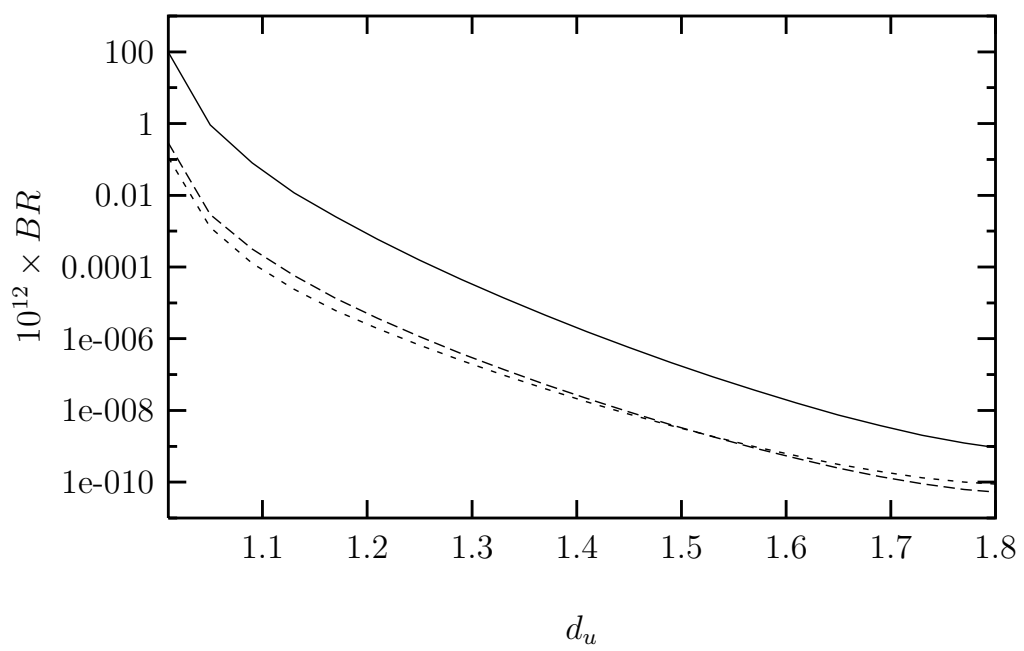

Figure 3: The scale parameter $d_{u}$ dependence of the BR $\left(r \rightarrow \mu^{ \pm} e^{ \pm}\right)$, for $\Lambda_{u}=10(\mathrm{TeV})$, the couplings $\lambda_{e e}=0.01, \lambda_{\mu \mu}=0.1$. The solid (dashed, small dashed) line represents the BR for $m_{r}=200(\mathrm{GeV})\left(m_{r}=500(\mathrm{GeV}), m_{r}=800(\mathrm{GeV})\right)$.

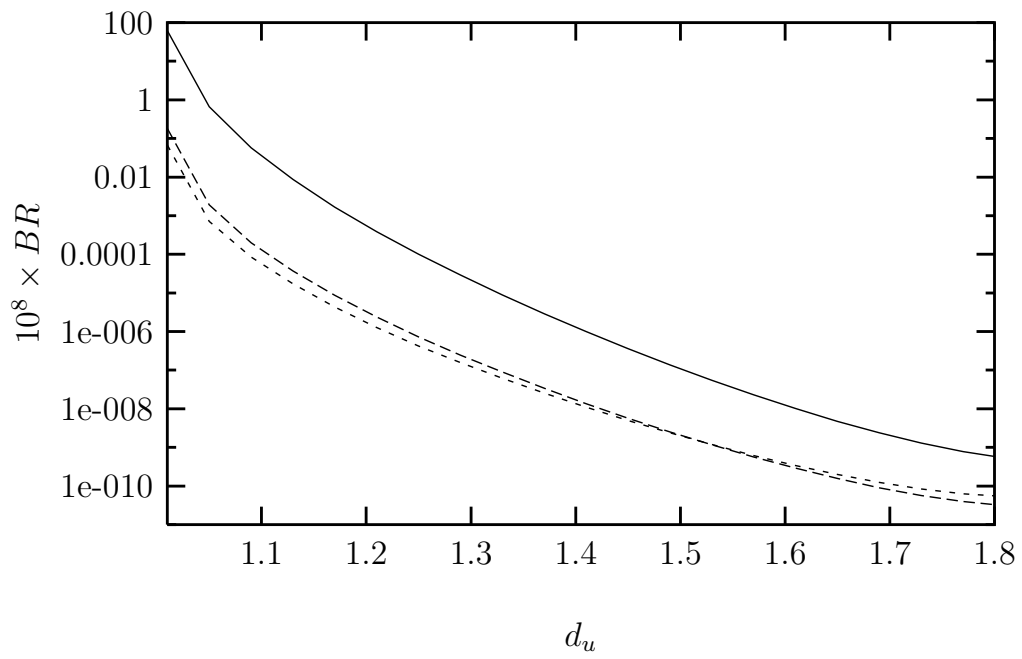

Figure 4: The scale parameter $d_{u}$ dependence of the $\mathrm{BR}\left(r \rightarrow \tau^{ \pm} e^{ \pm}\right)$, for $\Lambda_{u}=10(\mathrm{TeV})$, the couplings $\lambda_{e e}=0.01, \lambda_{\tau \tau}=1.0$. The solid (dashed, small dashed) line represents the BR for $m_{r}=200(\mathrm{GeV})\left(m_{r}=500(\mathrm{GeV}), m_{r}=800(\mathrm{GeV})\right)$. 


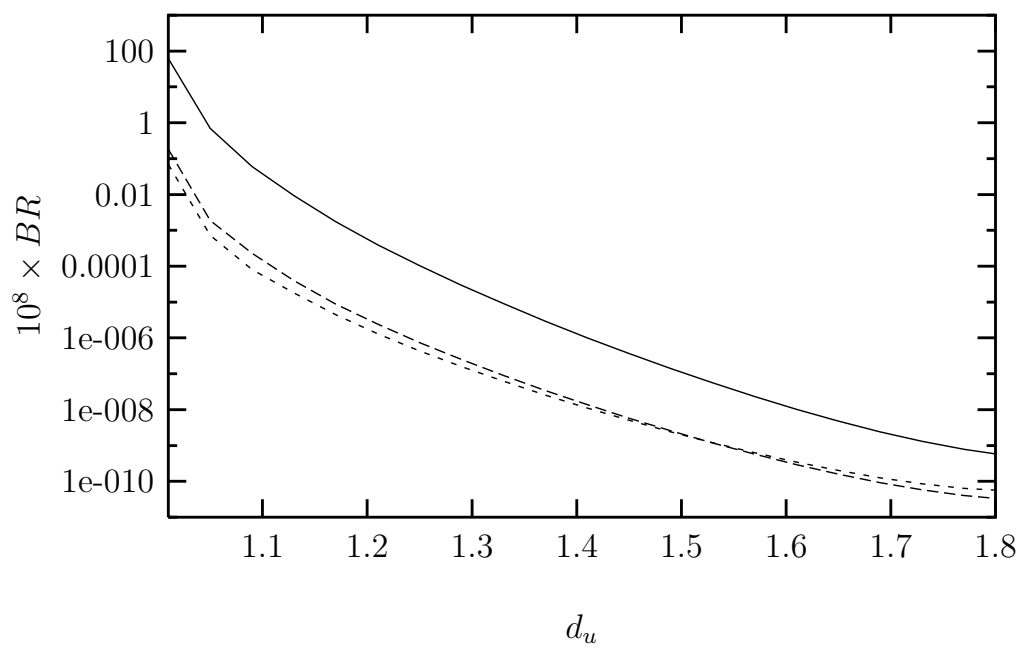

Figure 5: The scale parameter $d_{u}$ dependence of the BR $\left(r \rightarrow \tau^{ \pm} \mu^{ \pm}\right)$, for $\Lambda_{u}=10(\mathrm{TeV})$, the couplings $\lambda_{\mu \mu}=0.1, \lambda_{\tau \tau}=1.0$. The solid (dashed, small dashed) line represents the BR for $m_{r}=200(\mathrm{GeV})\left(m_{r}=500(\mathrm{GeV}), m_{r}=800(\mathrm{GeV})\right)$.

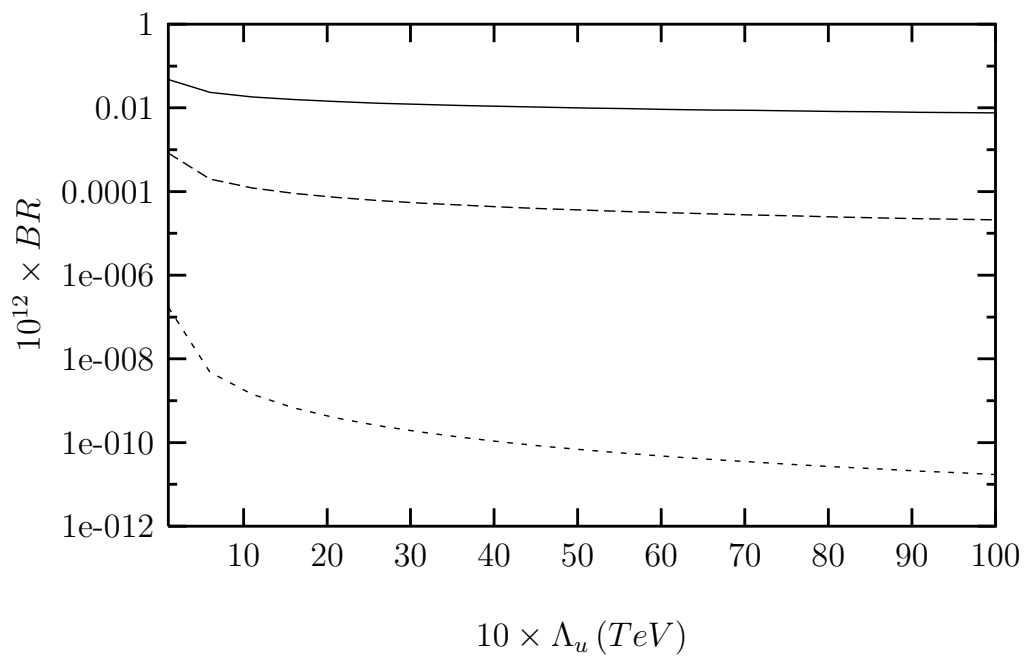

Figure 6: $\Lambda_{u}$ dependence of the BR $\left(r \rightarrow \mu^{ \pm} e^{ \pm}\right)$, for $m_{r}=200(\mathrm{GeV}), \lambda_{e e}=0.01$ and $\lambda_{\mu \mu}=0.1$. Here the solid-dashed-small dashed lines represent the BR for $d_{u}=1.1-1.2-1.5$. 


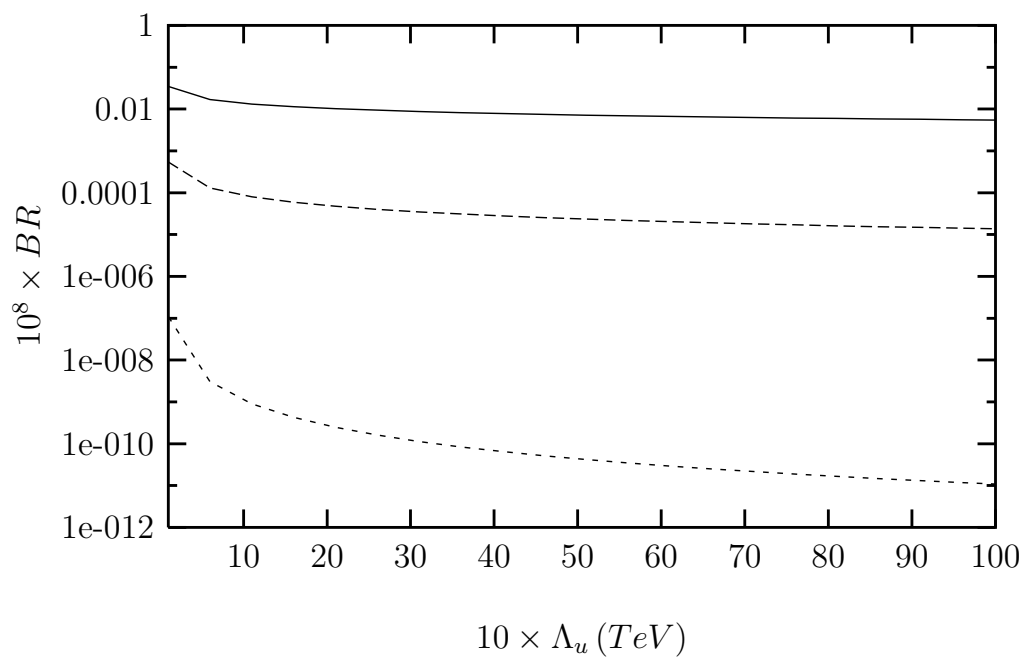

Figure 7: $\Lambda_{u}$ dependence of the BR $\left(r \rightarrow \tau^{ \pm} e^{ \pm}\right)$, for $m_{r}=200(\mathrm{GeV}), \lambda_{e e}=0.01$ and $\lambda_{\tau \tau}=1.0$. Here the solid-dashed-small dashed lines represent the BR for $d_{u}=1.1-1.2-1.5$.

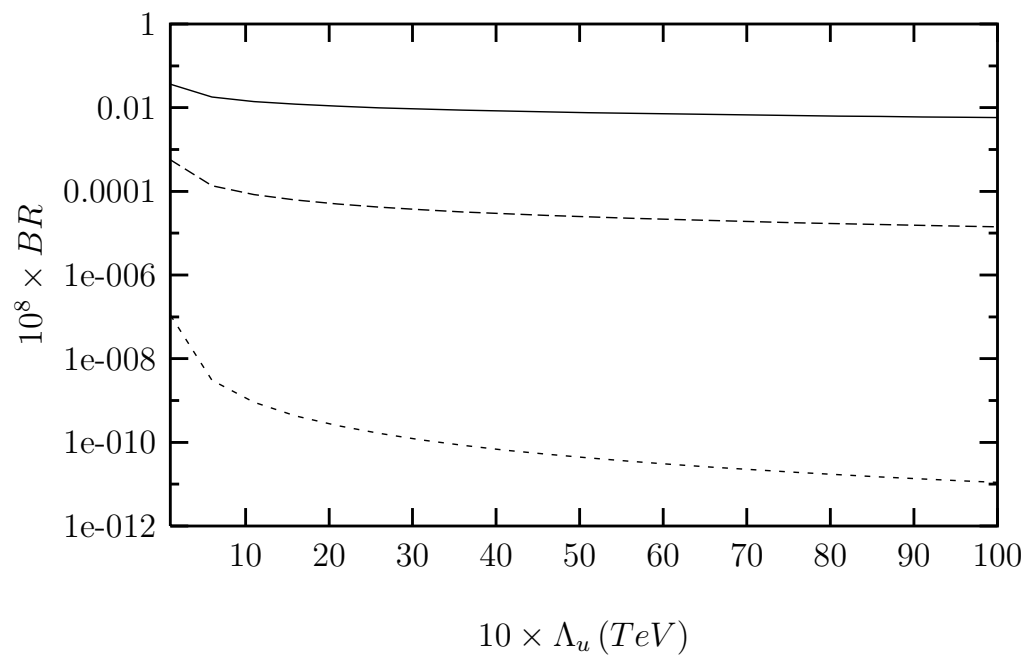

Figure 8: $\Lambda_{u}$ dependence of the BR $\left(r \rightarrow \tau^{ \pm} \mu^{ \pm}\right)$, for $m_{r}=200(\mathrm{GeV}), \lambda_{\mu \mu}=0.1$ and $\lambda_{\tau \tau}=1.0$. Here the solid-dashed-small dashed lines represent the BR for $d_{u}=1.1-1.2-1.5$. 


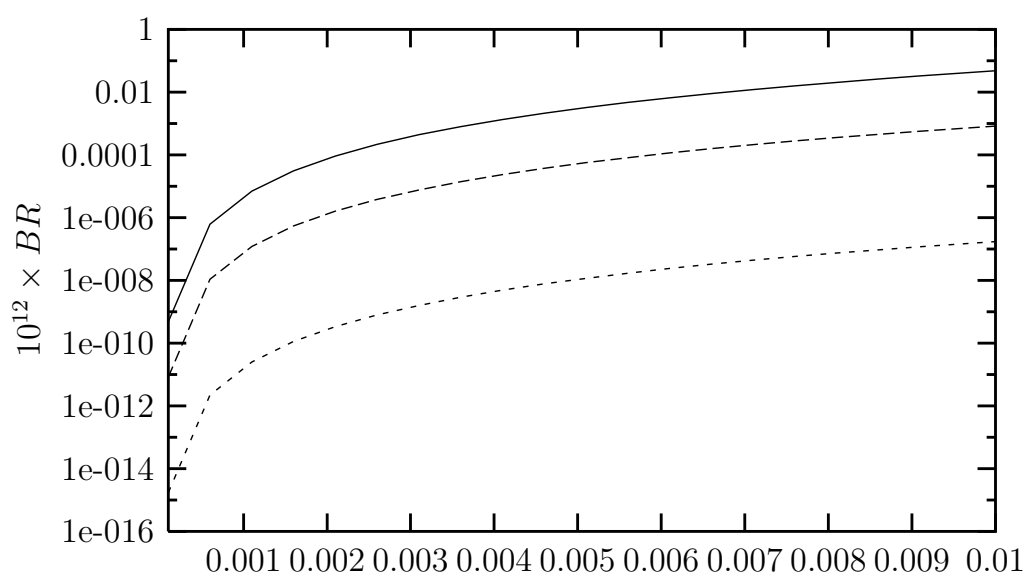

$\lambda$

Figure 9: $\lambda$ dependence of the BR $\left(r \rightarrow \mu^{ \pm} e^{ \pm}\right)$, for $m_{r}=200(\mathrm{GeV})$ and $\Lambda_{u}=10(T e V)$. Here the solid-dashed-small dashed lines represent the BR for $d_{u}=1.1-1.2-1.5$.

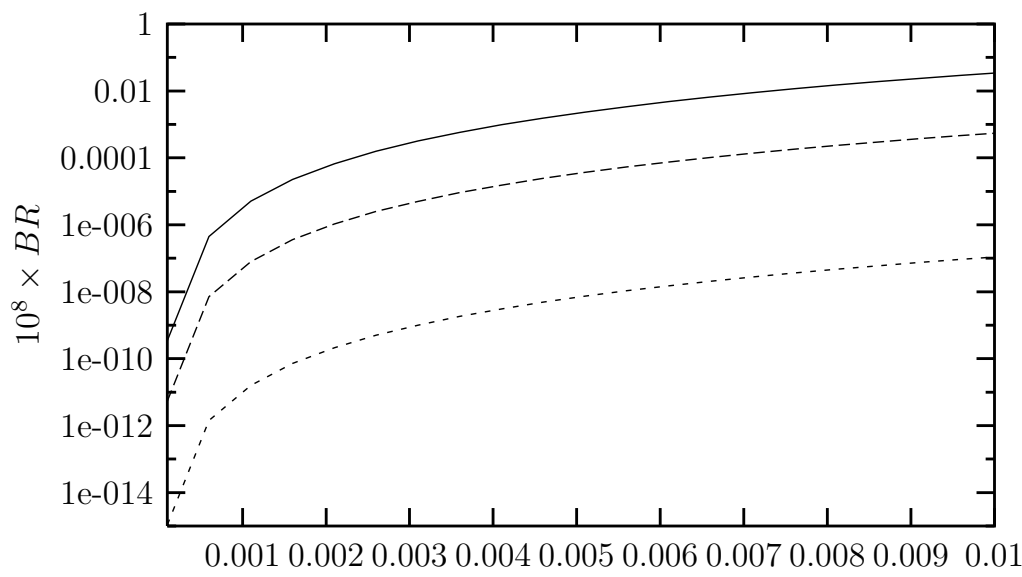

$\lambda$

Figure 10: $\lambda$ dependence of the $\operatorname{BR}\left(r \rightarrow \tau^{ \pm} e^{ \pm}\right)$, for $m_{r}=200(\mathrm{GeV})$ and $\Lambda_{u}=10(T e V)$. Here the solid-dashed-small dashed lines represent the BR for $d_{u}=1.1-1.2-1.5$. 


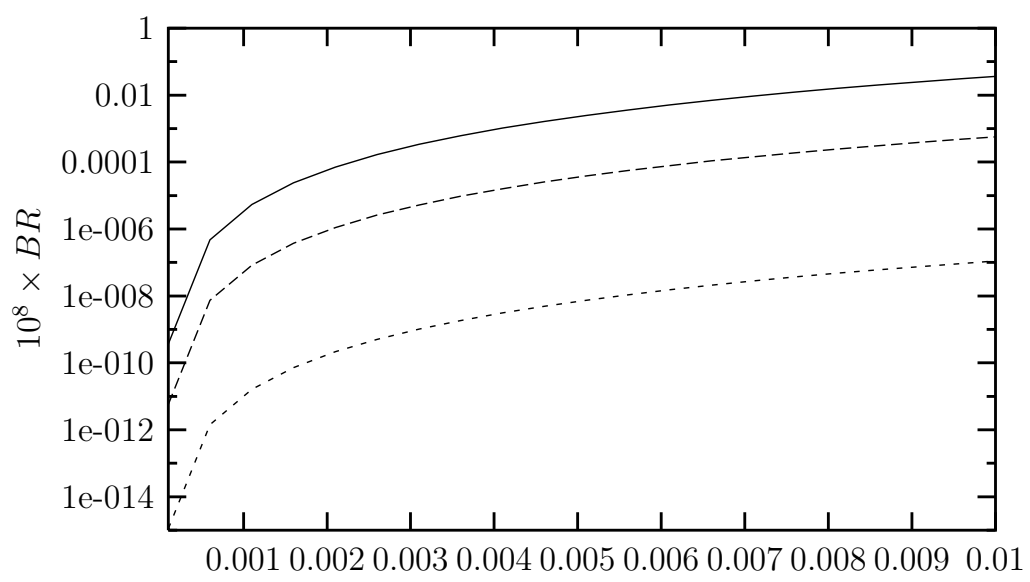

$\lambda$

Figure 11: $\lambda$ dependence of the BR $\left(r \rightarrow \tau^{ \pm} \mu^{ \pm}\right)$, for $m_{r}=200(\mathrm{GeV})$ and $\Lambda_{u}=10(\mathrm{TeV})$. Here the solid-dashed-small dashed lines represent the BR for $d_{u}=1.1-d_{u}=1.2-d_{u}=1.5$. 